\title{
The attractiveness of Plexiglas, a mirror, or an age mate to Peking ducklings and the relevance of these findings to imprinting experiments
}

\author{
L. JAMES SHAPIRO \\ Avian Behaviour Laboratory, University of Manitoba, Winnipeg R3T 2N2, Canada
}

\begin{abstract}
The attractiveness of Plexiglas, an age mate, and a mirror were evaluated by conducting a series of preference tests for these objects with white Peking ducklings. The live age mate was preferred more than the other two objects. When the live age mate was not present, the subject's image in Plexiglas was preferred significantly more than the same image in a mirror or an empty quadrant. When presented by itself, the subject's image in Plexiglas was preferred significantly more than three empty quadrants. The relevance of these results for imprinting experiments utilizing a Plexiglas maze is discussed.
\end{abstract}

Imprinting experiments frequently evaluate the strength of a young subject's attachment to some object by measuring the degree to which the subject follows the more familiar of two objects presented to it. One of the earlier experiments involving this phenomenon was done by Hess (1959) and involved a circular Plexiglas maze. Several years ago, I constructed a similar apparatus to evaluate the strength of the following response in young chicks and ducklings (Shapiro, 1970). Subsequently, subjects were frequently seen in close proximity to one of the stimulus objects, but at the same time, the subjects seemed to be looking into the Plexiglas.

In the imprinting paradigm mentioned above, it is assumed that a strong following response indicates that a strong attachment has been formed, whereas a weak following response, presumably, means that a weaker bond has been formed.

It seems to me, however, that the latter situation would be true only if there were no distracting stimuli encouraging the subject to look elsewhere and, perhaps, to approach something in addition to or instead of the "correct" stimulus object. In fact, it was observed that frequently the subjects would spend more time orienting to the sides of the maze than they would to the stimulus objects. It is likely that if the stimulus object used in an imprinting experiment is inappropriate to the subject

These experiments were conducted while the author was a postdoctoral fellow under the sponsorship of J. P. Scott at Bowling Green State University. The studies were subsequently analyzed and revised at the author's present address. The author wishes to thank Dr. Scott for his financial support and encouragement in conducting these experiments. I also wish to thank Belinda Hopkins for running the subjects used in these studies. Requests for reprints should be sent to L. James Shapiro, Avian Behaviour Laboratory, Department of Psychology, University of Manitoba, Winnipeg, Manitoba R3T 2N2, Canada. in the first place, then the subject will probably pay more attention to a more attractive (and consequently distracting) stimulus. One possible candidate for such a stimulus seemed to be the subject's image in the Plexiglas used to construct the circular maze. The image of another duckling may be a much more attractive stimulus object than an inanimate, possibly inappropriate, stimulus object. The purpose of these experiments, then, was to evaluate a subject's preference for its own image or similar variations of it.

\section{GENERAL METHOD}

\section{Subjects and Procedure}

Three experiments were conducted in which 120 white Peking ducklings (Anas platyrhynchos) were used. The subjects were communally hatched in the dark in large hatchers at the Wayne Duck Farm in Wayne, Ohio, and were individually selected by the experimenter. It was estimated that the subjects were no more than 4 or $5 \mathrm{~h}$ of age when they were picked up. The procedure used in selecting subjects is described in Shapiro (1977). The ducklings were transported communally, in the dark, to the aviary at Bowling Green State University. Here they were randomly placed in specially constructed brood units (Shapiro \& Lundy, 1974) that allowed subjects to be individually housed in the dark and automatically fed and watered without ever being exposed to anything other than their own sounds and the sounds of adjacent birds. The temperature of the aviary was maintained at $28.3^{\circ} \mathrm{C}$.

When a subject was to be used, it was removed from its cage, placed into a transport box, and carried to the imprinting laboratory (Shapiro, 1970). Inside the imprinting laboratory, the subjects were placed, in the dark, at the center of a sandcovered table that was inside a temperature-controlled, sounddeadened experimental chamber. In each corner of this table, a stimulus object could be located. The subjects were always placed so that they faced an empty quadrant.

When the experimenter left the experimental chamber, he placed himself in front of a one-way window, turned on the lights inside the chamber, and observed the behavior of each duckling for $15 \mathrm{~min}$. The amount of time each subject spent in 
each quadrant was recorded by means of a stopwatch. At the end of each trial, the lights were turned off and the subject was removed from the experimental chamber. The subject was not used again in the experiment. The temperature of the chamber was maintained around $23.8^{\circ} \mathrm{C}$

The subjects in the first group of each experiment were approximately $15-18 \mathrm{~h}$ old when they were tested. This age range will be called Day 1 . Each successive group of subjects was then run $24 \mathrm{~h}$ following the preceding group. Day 2, therefore, represents subjects approximately $39-42 \mathrm{~h}$ old. Forty subjects were used in each experiment. Eight different subjects were tested individually each day for 5 days. Eight subjects per day is a large sample size with respect to the typical imprinting study reported in the literature.

\section{Statistical Analysis}

A two-way analysis of variance with repeated measures on one factor was used to analyze the data from each experiment. When significant interaction terms were present, a simple-effects analysis of variance and the Newman-Keuls procedure were utilized. The Newman-Keuls procedure was also used to analyze significant main effects.

\section{EXPERIMENT 1: LIVE AGE MATE, PLEXIGLAS, MIRROR}

In the first experiment, three stimulus objects were used. One stimulus object was an age mate of the subject. Another stimulus object was a concave piece of the Plexiglas used to construct the circular maze. Black cloth lined the back of the Plexiglas and prevented the subjects from seeing through it, while at the same time increasing the reflectiveness of the Plexiglas. The third stimulus object was a mirror. The age mates used as stimulus objects were habituated to the experimental chamber and to their holding cages prior to each day's testing session. They rarely vocalized during a testing session.

\section{Results}

The results of the first experiment are illustrated in Figure 1. There was a significant $(p<.05)$ Days by Stimulus object interaction. An analysis of the simple

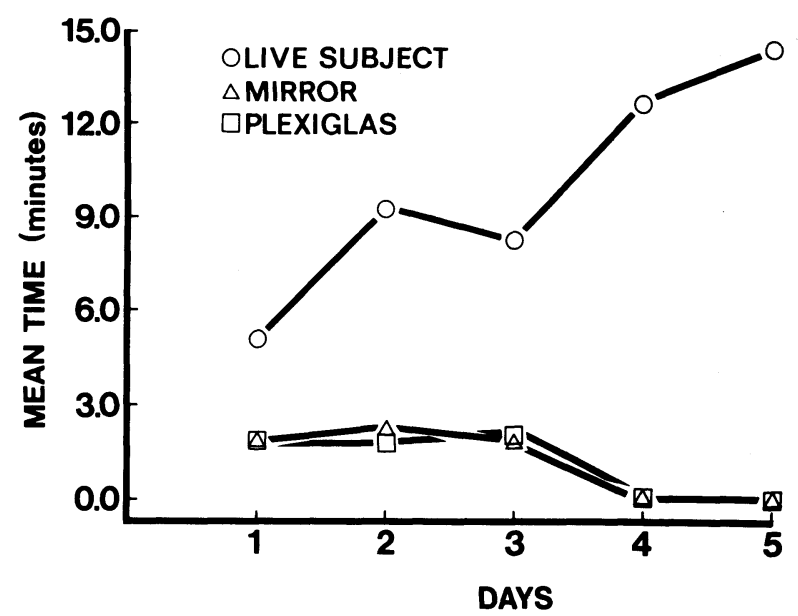

Figure 1. Mean time spent near a live age mate, a mirror, or a Plexiglas stimulus object.

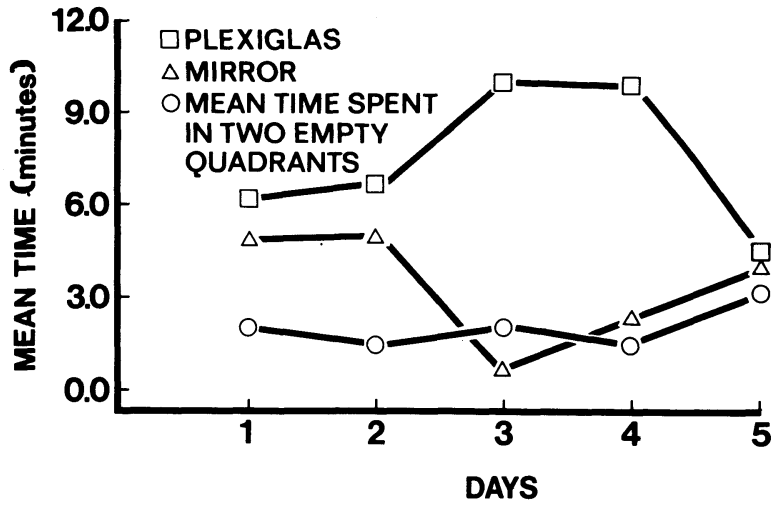

Figure 2. Mean time spent near a Plexiglas stimulus object, a mirror, or in two empty quadrants.

effects indicated that the live age mate was preferred significantly more $(p<.05)$ than the mirror image or the Plexiglas image on all days except Day 1. On Day 1 the subjects did not prefer the live age mate significantly more than the mirror or the piece of Plexiglas. The live age mate was highly attractive to them, however, since the subjects were in its vicinity 2.5 times more than they were in the vicinity of the other two stimulus objects. The Plexiglas image was preferred almost exactly as much as the mirror image.

\section{EXPERIMENT 2: PLEXIGLAS, MIRROR, TWO EMPTY QUADRANTS}

In the second experiment, the live age mate was removed and the piece of Plexiglas and the mirror were retained. Each stimulus object appeared in each quadrant an equal number of times.

\section{Results}

The results of the second experiment are illustrated in Figure 2. There was a significant $(p<.01)$ main effect attributable to stimulus objects. No other component of the analysis was significant. An analysis of the main effects revealed that the Plexiglas image was preferred significantly more $(p<.01)$ than either the mirror image or the empty quadrants.

\section{EXPERIMENT 3: PLEXIGLAS PLUS THREE EMPTY QUADRANTS}

In the third experiment, only the piece of Plexiglas remained as a stimulus object. It was placed in each quadrant an equal number of times. In those imprinting experiments in which there is a Plexiglas maze, there is usually no other source of obvious stimulation other than the walls of the Plexiglas maze and the stimulus object or objects. The third experiment, therefore, approximated the conditions found in an imprinting experiment utilizing a Plexiglas maze more so than did Experiments 1 and 2. 


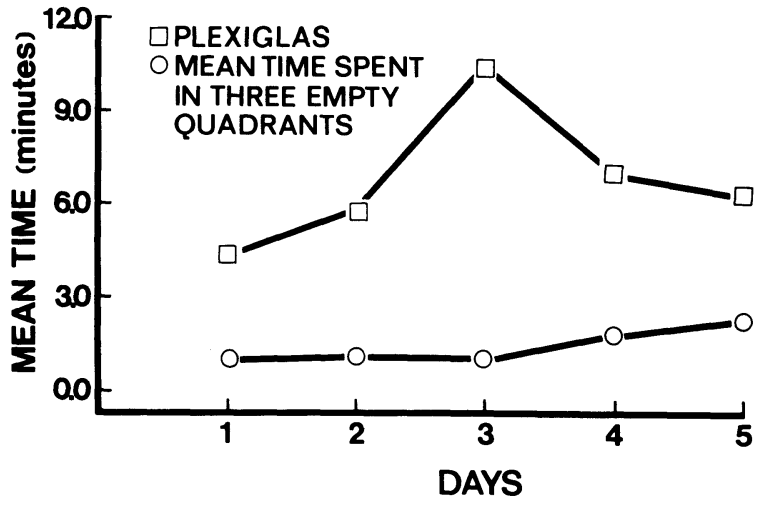

Figure 3. Mean time spent near a Plexiglas stimulus object or in three empty quadrants.

\section{Results}

The results of this experiment are presented in Figure 3. There was a significant $(p<.01)$ main effect attributable to the stimulus object. The piece of Plexiglas was preferred significantly more $(p<.01)$ than the empty quadrants.

\section{GENERAL DISCUSSION}

The results of these experiments indicate that if a Plexiglas maze is used in an imprinting experiment, then the experimenter should at least be aware of the potentially biasing effect of the Plexiglas used to construct the maze.

In such a situation, the investigator would be presenting at least two attractive choices to the subject during a testing trial. One object would be the subject's own reflection in the walls of the Plexiglas maze, and the other object would presumably be the more familiar of the two objects that the experimenter chose to present to the subject.

One cannot assume that the subject's reflection is unfamiliar to it and consequently unattractive to it. For one thing, the subject would see its reflection during its training trial, when the experimenter was exposing the subject to the object that would later be said to be "more familiar" to it. In addition, if the subject is reared in isolation but in an illuminated area, it can still see parts of its own body, and those parts that it does see may assist it in forming a bond to its species (Shapiro, 1980). The subject's reflection in a piece of Plexiglas would also contain those aspects of its body that it could see if it was reared in isolation.

Hence, the subject's reflection cannot be considered an "unfamiliar" object at all. During a testing trial, then, the subject must choose between three stimulus objects, two of which are familiar and presumably attractive and one of which is unfamiliar (but which may also have attractive components to it, unknown to the experimenter). Given such a situation, the subject finds itself in an approach-approach conflict. Such conflicts are characterized by ambivalence, high latency times, and the absence of a clear-cut decision on the part of the subject. The performance of a duckling may also be described in this way, in this situation.

The above discussion assumes that all stimulus objects are equally attractive to a duckling but that more familiar objects are more attractive. This assumption may not be true at all. There may very well be a continuum of stimuli that can be rated in terms of their biological importance to the duckling and are treated as such on their very first presentation to the subject (see, for example, Storey \& Shapiro, 1979). Hence, a biologically important stimulus may be more attractive to a subject on its first presentation than a less important, biologically inappropriate stimulus that has been presented to the subject one or more times in the past. If a subject's reflection falls into such a category, then it could potentially be a much more attractive stimulus to a duckling than the more familiar object being presented to it during the testing trial or the less familiar object being presented to it as an alternative to the more familiar object.

Support for this position can be found in Experiment 1, in which the subjects exhibited a preference for a live age mate. This preference illustrated the unequal attractiveness of "similar" stimuli presented to a subject for the first time. The more biologically appropriate stimulus was preferred. (Since vocalizations were rarely emitted by the age mates used as stimulus objects, vocalizations cannot be cited as an important component in eliciting an approach response from the subjects being tested in this situation, although vocalization is an important component in the formation of an attachment in ducklings.)

If the stimulus object used as a maternal surrogate in an imprinting experiment is not attractive to subjects, then the subjects may spend more time near their reflected images than they do in following the maternal surrogate. The results of Experiments 2 and 3 illustrate the attractiveness of the subject's reflection in a piece of Plexiglas. Conversely, if the "more familiar" stimulus object is very effective in eliciting a following response, then the subject's reflected image will probably not be preferred. In fact, the reflection of the more preferred stimulus object in the Plexiglas may provide the subject with "two" of this preferred object. Rightly or wrongly, the experimenter is providing more of this stimulus than was originally thought to be the case.

As the stimulus objects used in imprinting experiments become more and more inappropriate for use as maternal surrogates, the confounding exerted by a Plexiglas maze will probably exert its influence more and more. In terms of evaluating the strength of a subject's preference for the investigator's choice of a maternal surrogate, the investigator may assume that it is weak when, in fact, it may be very strong. In the former case, the subject may not be forming a very strong attachment to the inappropriate maternal surrogate. In the latter case, the subject may be forming a stronger attachment to the more attractive and more appropriate stimulus reflected in the Plexiglas.

\section{REFERENCES}

Hess, E. Imprinting. Science, 1959, 130, 133-141.

Shapiro, L. J. Automation and experimental control in a laboratory for imprinting research. Journal of Comparative and Physiological Psychology, 1970, 73, 421-426.

Shapiro, L. J. Developing preferences for live female models of the same or other species in white Peking ducklings. Animal Behaviour, 1977, 25, 849-858.

Shapiro, L. J. Species identification in birds: A review and synthesis. In A. Roy (Ed.), Species identity and attachment. New York: Garland, 1980.

Shapiro, L. J., \& Lundy, C. R. Brood units for rearing and maintaining birds communally or in isolation. Journal of Biological Psychology, 1974, 16, 40-44.

Storey, A. E., \& Shapiro, L. J. Development of preferences in white Peking ducklings for stimuli in the natural post-hatch environment. Animal Behaviour, 1979, 27, 411-416.

(Received for publication May 8, 1980.) 